\title{
Cultura de segurança do paciente: perspectiva de trabalhadores da saúde e apoio
}

Patient safety culture: perspective of health and support workers

Cultura de seguridad del paciente: perspectiva de trabajadores de la salud y de apoyo

Mari Angela Meneghetti Baratto ${ }^{1}$ (D) https://orcid.org/0000-0002-2635-0725

Enio Júnior Seidel' ${ }^{1}$ ๑ https://orcid.org/0000-0002-9656-0699

Bruna Xavier Morais ${ }^{1}$ i nttps://orcid.orgy/0000-0002-0446--9231

Oclaris Lopes Munhoz ${ }^{1}$ ib hitps://orcid.org/0000-0001--8901-7148

Silvana Cruz da Silva² io https://oridi.org/0000-0002-4563-3704

Caroline Zottele ${ }^{1}$ io https:///orcid.org/0000-0002-4537--399x

Tania Solange Bosi de Souza Magnago ${ }^{1}$ io https://orcid.org/0000-0002-5308-1604

Baratto MA, Seidel EJ, Morais BXmo citar: Silva SC, Zottele C, et al. Cultura de segurança do paciente: perspectiva de trabalhadores da saúde e apoio. Acta Paul Enferm. 2021;34:eAPE001595.

DOI http://dx.doi.org/10.37689/actaape/2021A0001595

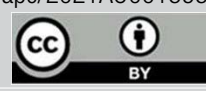

Descritores Segurança uo paciente; Atitude do pessoal de saúde; Cultura organizacional; Pessoal de saúde; Gestão da segurança

Keywords

Patient safety; Attitude of health personnel; Organizational culture; Health personnel; Safety management

Descriptores Seguridad del paciente; Actitud del personal de salud; Cultura organizacional; Personal de salud; Administración de la seguridad

Submetido 22 de Junho de 2020 Aceito 2 de Dezembro de 2020

Autor correspondente Tania Solange Bosi de Souza Magnago E-mail: magnago.tania@gmail.com

\section{Resumo}

Objetivo: Analisar a cultura de segurança do paciente na perspectiva dos trabalhadores que atuam direta ou indiretamente no cuidado ao paciente hospitalizado.

Métodos: Estudo transversal, com 2.634 trabalhadores do serviço hospitalar de sete instituições do Rio Grande do Sul, Brasil. Utilizou-se a versão brasileira do Safety Attitudes Questionnaire. Realizaram-se análises descritiva e inferencial, considerando cultura positiva escore $\geq 75$ pontos.

Resultados: Evidenciou-se avaliação positiva da cultura de segurança nos domínios Clima de trabalho em equipe (mediana 75) e Satisfação no Trabalho (mediana 90). Os fisioterapeutas, dentistas e trabalhadores da manutenção avaliaram de forma positiva a cultura de segurança $(p<0,05)$. Psicólogos, profissionais da nutrição/dietética e vigilantes/porteiros tiveram maiores percentuais para cultura negativa $(p<0,05)$.

Conclusão: A cultura de segurança obteve escores predominantemente negativos, mais expressivos no domínio percepção da gerência do hospital. Quando comparadas as categorias da saúde e apoio, identificouse pouca variabilidade nos escores dos domínios do instrumento. No entanto, os profissionais do apoio tenderam a pontuações mais baixas. Avaliar as dimensões da cultura de segurança fornece um diagnóstico situacional da organização ou unidade de trabalho e pode subsidiar estratégias gerenciais com vistas ao aprimoramento da qualidade da assistência prestada ao paciente.

\section{Abstract}

Objective: To analyze the culture of patient safety from the perspective of workers working directly or indirectly in the care of hospitalized patients.

Methods: Cross-sectional study of 2,634 hospital service workers from seven institutions in Rio Grande do Sul, Brazil. The Brazilian version of the Safety Attitudes Questionnaire was used. Descriptive and inferential analyzes were performed, considering scores $\geq 75$ points as positive culture.

Results: A positive evaluation of the safety culture was evidenced in the Teamwork climate (median 75) and Job Satisfaction (median 90) domains. Physiotherapists, dentists and maintenance workers evaluated the safety culture positively $(p<0.05)$. Psychologists, nutrition/dietetics professionals and security guards/ doormen achieved higher percentages for negative culture $(p<0.05)$.

Conclusion: The safety culture obtained predominantly negative scores, more expressive in the Perception of hospital management domain. When comparing the health and support categories, little variability was identified in scores of the instrument domains, although support professionals tended to score lower. Assessing the dimensions of the safety culture provides a situational diagnosis of the organization or work unit and can support management strategies aimed at improving the quality of patient care.

${ }^{1}$ Universidade Federal de Santa Maria, Santa Maria, RS, Brasil.

¿Universidade Franciscana, Santa Maria, RS, Brasil.

Conflitos de interesse: extraído da dissertação: "Cultura de segurança do paciente: percepções e atitudes de trabalhadores nas instituições hospitalares de Santa Maria“, Programa de Pós-Graduação em Enfermagem, Universidade Federal de Santa Maria, 2015. 


\section{Resumen}

Objetivo: Analizar la cultura de seguridad del paciente desde la perspectiva de los trabajadores que actúan directa o indirectamente en el cuidado al paciente hospitalizado.

Métodos: Estudio transversal con 2.634 trabajadores del servicio hospitalario de siete instituciones del estado de Rio Grande do Sul, Brasil. Se utilizó la versión brasileña del Safety Attitudes Questionnaire. Se realizó un análisis descriptivo e inferencial y se consideró como cultura positiva la puntuación $\geq 75$.

Resultados: Se observó una evaluación positiva de la cultura de seguridad en los dominios Clima de trabajo en equipo (mediana 75) y Satisfacción en el trabajo (mediana 90). Los fisioterapeutas, dentistas y trabajadores de mantenimiento evaluaron de forma positiva la cultura de seguridad ( $<<0,05)$. Los psicólogos, profesionales de nutrición/dietética y vigilantes/porteros tuvieron porcentajes mayores de cultura negativa $(p<0,05)$.

Conclusión: La cultura de seguridad obtuvo puntuaciones predominantemente negativas, más significativas en el dominio Percepción de la gerencia del hospital. Al comparar las categorías de salud y de apoyo, se identificó poca variabilidad en las puntuaciones de los dominios del instrumento. Sin embargo, los profesionales de apoyo tuvieron una tendencia de puntajes más bajos. Evaluar las dimensiones de seguridad ofrece un diagnóstico situacional de la organización o unidad de trabajo y puede respaldar estrategias gerenciales con el fin de mejorar la calidad de la atención prestada al paciente.

\section{Introdução}

A promoção do cuidado seguro nas organizaçóes de saúde é essencial para a qualidade assistencial prestada aos pacientes. A falta de segurança no ambiente de trabalho pode repercutir negativamente na vida dos profissionais, gerar ônus social e financeiro, desestabilizar os processos laborais, além de repercutir em publicidade negativa aos envolvidos. ${ }^{(1)}$

Os serviços de saúde, pela complexidade das demandas, são considerados de alto risco para ocorrência de incidentes. $\mathrm{O}$ desenvolvimento de uma cultura de segurança é fundamental e exige o engajamento de todos. ${ }^{(1,2)}$ Conceitualmente, é entendida como um "conjunto de valores, atitudes, competências e comportamentos que determinam o comprometimento com a gestáo da saúde e da segurança, substituindo a culpa e a punição pela oportunidade de aprender com as falhas e melhorar a atenção à saúde". ${ }^{(2,3)}$ Assim, a promoção de uma cultura justa favorece a comunicação efetiva, o trabalho em equipe e a transmissão do conhecimento, contribuindo para práticas assistenciais exitosas.

A avaliação da cultura de segurança institucional resulta do clima que envolve o ambiente de uma organização e como este é percebido pelos trabalhadores. Nela, é possível identificar as características de uma organização; quais são as suas pretensôes e o quanto a sua gestão é engajada para trabalhar com as potencialidades e fragilidades. Os componentes mensuráveis da cultura de segurança, são os comportamentos observados, as políticas, as práticas, os procedimentos e as percepçóes dos profissionais. ${ }^{(2,4)}$ Pesquisa $^{(1)}$ realizada com 1.342 colaboradores de 32 organizaçóes apontou que, nas instituiçóes com cultura positivas, os requisitos de segurança tendem a ser desempenhados pelos funcionários, independente da fiscalização do supervisor. ${ }^{(1)}$ Tal evidência demonstra a importância de fortalecê-la cotidianamente.

Uma das maneiras de mensurar a cultura de segurança é por meio do Safety Attitudes Questionare (SAQ), traduzido e validado para a língua portuguesa. $^{\text {(2) }} \mathrm{O}$ SAQ possui 41 questóes dispostas em seis domínios, que focalizam as atitudes e percepçóes dos trabalhadores de diferentes serviços e equipes profissionais. A utilização deste instrumento possibilita a realização de comparações. ${ }^{(2,5)}$

Neste tocante, existe uma preocupação crescente das organizaçôes de saúde em mapearem a cultura de segurança do paciente para balizar a tomada de decisão direcionada aos pontos frágeis identificados. Todavia, os estudos ${ }^{(4,6,7)}$ tem pesquisado basicamente profissionais da saúde. Entretanto, apesar de existirem microculturas locais em uma mesma instituição, ${ }^{(8)}$ essa temática perpassa todas as áreas. Ou seja, envolve todos os profissionais que nela atuam, direta ou indiretamente, no cuidado ao paciente. Com isso, o foco da avaliação com categorias da mesma área, sinaliza para uma lacuna no conhecimento direcionada ao diagnóstico da cultura de segurança na ótica dos demais trabalhadores; por exemplo, daqueles que compóem os serviços de apoio. Nesse contexto, objetivou-se analisar a cultura de segurança do paciente na perspectiva dos trabalhadores que atuam direta ou indiretamente no cuidado ao paciente hospitalizado. 


\section{Métodos}

Estudo transversal, desenvolvido em sete instituiçóes hospitalares de pequeno, médio e grande porte, localizadas na regiáo central do Rio Grande do Sul, Brasil. A população foi constituída por todas as categorias de profissionais da área da saúde (enfermeiro, auxiliar/técnico de enfermagem, médico, farmacêutico, fisioterapeuta, nutricionista, psicólogo, dentista) e do serviço de apoio (chefias/diretores, administrativo, higiene e limpeza, nutrição/dietética, manutençấo, vigilante porteiro), independente da unidade ou setor de atuação, totalizando 4.040 trabalhadores. A amostra mínima de participantes foi calculada, tendo-se por base um percentual estimado de $50 \%$ e um erro amostral de $1 \%$, o que resultou em 2.508 participantes.

O critério de inclusão foi o de estarem atuando há pelo menos 30 dias na instituição, período necessário para vivenciarem a cultura de segurança organizacional. ${ }^{(9)}$ Excluiram-se os trabalhadores em licença médica ou afastados por qualquer razáo durante a coleta de dados. Nesse critério, 648 (16\%) trabalhadores foram excluídos, totalizando uma populaçáo elegível de 3.392. Destes, foram consideradas perdas: 694 (20,5\%) (recusas; não serem encontrados no dia e horário da coleta no setor, após três tentativas; indisponibilidade de tempo para preencher o instrumento) e 64 $(1,89 \%)$ por exclusão de questionários incompletos. Assim, 2.634 trabalhadores participaram do estudo.

A coleta dos dados ocorreu entre fevereiro e agosto de 2014, após autorizaçáo institucional e tramitação no Comitê de Ética em Pesquisa. O convite para participar do estudo foi realizado pela pesquisadora, durante as reunióes de equipe e em abordagens individuais. A aplicação dos questionários foi realizada por 18 auxiliares de pesquisa, previamente capacitados pela coordenadora do projeto. Os instrumentos foram respondidos individualmente no próprio local de trabalho, em espaço reservado, após a assinatura do Termo de Consentimento Livre e Esclarecido, em duas vias. Forneceram-se todos os esclarecimentos necessários, respeitando a Resolução 466/12, do Conselho Nacional de Saúde.

Como protocolo de pesquisa, utilizou-se a versáo brasileira do SAQ.(2) Instrumento com 41 questôes distribuídas em seis domínios da cultura de segurança do paciente (clima de trabalho em equipe; Clima de segurança; satisfaçáo no trabalho; reconhecimento do estresse; percepção da gestão da unidade e do hospital e condiçôes de trabalho). As opçóes de respostas seguem uma escala likert de cinco pontos: discordo totalmente (A), discordo parcialmente (B), neutro (C), concordo parcialmente (D), concordo totalmente (E) e não se aplica $(\mathrm{X}){ }^{(2)} \mathrm{O}$ SAQ contempla uma segunda parte, composta por dados demográficos e laborais (sexo, profissão, tempo de atuação na especialidade e atuaçáo principal). Além desses, foram acrescidas: idade, turno de trabalho, outro emprego, horas extras e atuaçáo direta ou indireta com o paciente.

Os dados foram organizados no programa Epiinfo ${ }^{\circ}$, versão 6.4 , com dupla digitação independente. Após a verificação de erros e inconsistências da digitação, a análise dos dados foi realizada no software $R^{\circ}$. As variáveis categóricas foram analisadas por meio das frequências absoluta (n) e relativa (\%). As variáveis quantitativas foram expressas pelas medidas de tendência central e de dispersão, de acordo com a distribuiçấo de normalidade ou não dos dados, avaliados pelo teste Kolmogorov-Smirnov. A análise da confiabilidade do SAQ foi realizada por meio do Alfa de Cronbach.

A análise da cultura de segurança foi realizada pelo escore geral do SAQ e por seus domínios. A pontuação pode variar de 0 a 100 pontos, sendo zero a pior percepção do clima de segurança e 100 a melhor percepção. A dicotomização em "Alto e Baixo" foi realizada utilizando o ponto de corte indicado para cultura de segurança positiva ( $\geq 75$ pontos). ${ }^{(2)}$ Após a inversão dos itens reversos (itens 2, 11 e 36), a análise descritiva do SAQ foi realizada pela média das respostas aos seus 41 itens, conforme recomendam as autoras. ${ }^{(2)} \mathrm{Na}$ sequência, os itens foram agrupados por domínios, calculando-se o escore do domínio, por meio da fórmula $(\mathrm{m}-1) \times 25$, na qual $m$ é a média dos itens de cada domínio $[0-100]{ }^{(2)}$

Para a análise das categorias profissionais, foram realizadas avaliaçôes conjuntas (profissionais $\mathrm{da}$ saúde e dos serviços de apoio) e distintas por categorias profissionais. Utilizaram-se as seguintes terminologias na descrição dos resultados: Geral (Saúde e Apoio), Saúde (profissionais da saúde) e Apoio 
(demais profissionais da instituição: serviços de higiene e limpeza, manutenção, nutrição e dietética e administrativos, como almoxarifado, secretaria e vigilância). Para a análise bivariada entre os domínios do SAQ e a categoria profissional utilizou-se o teste $U$ de Mann-Whitney e Qui-quadrado. Em todas as análises, adotou-se o nível de significância de 5\%.

Esta pesquisa obteve aprovação por Comitê de Ética em Pesquisa, sob o parecer no 494.080 e CAAE no 25325613.5.0000.5346.

\section{Resultados}

Obteve-se uma taxa geral de respostas de 77,7\%, sendo que as instituiçóes de pequeno e médio porte apresentaram melhor adesão à pesquisa. Participaram 1.830 (69,5\%) profissionais da saúde e 804 (30,5\%), do serviço de apoio. Predominaram trabalhadores $(\mathrm{n}=1.901 ; 72,6 \%)$ do sexo feminino; com idade entre 19 e 38 anos $(\mathrm{n}=1.312 ; 50,9 \%)$; atuantes em turnos mistos $(n=1.182 ; 45,1 \%)$ e que prestavam cuidados direto aos pacientes $(n=1.854$; $71,6 \%)$. Prevaleceram os trabalhadores que não exerciam cargo de chefia $(n=2.412 ; 91,9 \%)$, que não possuíam outro emprego $(n=2.097 ; 79,9 \%)$ e que não realizavam horas extras $(\mathrm{n}=1.727 ; 66,2 \%)$.

$\mathrm{Na}$ tabela 1, estão apresentadas as análises descritivas das pontuaçóes do SAQ total e de seus domínios; bem como, a consistência interna do instrumento de acordo com as categorias profissionais pesquisadas.

A cultura de segurança foi negativa (mediana $70,1)$, com avaliação positiva nos domínios “Satisfação no trabalho" e "Clima de trabalho em equipe" (mediana $\geq 75$ ). Evidenciou-se que o domínio "Percepção de gerência do hospital", obteve a menor pontuação. O SAQ apresentou consistência interna adequada $(\alpha=0,90)$ na avaliação da categoria Geral, bem como na aferição individualizada por categoria Saúde e Apoio $(\alpha=91)$. A avaliação da cultura de segurança, por categoria profissional e discriminada entre os seis domínios está apresentada na tabela 2 .

$\mathrm{Na}$ avaliação por categorias, o escore positivo para cultura de segurança foi alcançado nos domínios "Clima de trabalho em equipe" e "Satisfação no trabalho" pelos profissionais da saúde e somente no domínio "Satisfação no trabalho" pelos de apoio. A tabela 3 apresenta a avaliação da cultura de segurança, de acordo com os domínios do SAQ e categorias profissionais.

Dentre os trabalhadores da saúde, os fisioterapeutas tiveram melhor avaliação positiva para "Clima de trabalho em equipe" $(\mathrm{p}=0,026)$, "Clima de segurança” ( $p=0,012)$; os dentistas para "Satisfação no trabalho" ( $\mathrm{p}=0,025)$; já os psicólogos apresentaram as mais baixas avaliaçóes para "Condiçóes de trabalho” ( $\mathrm{p}=0,001)$. Quanto à categoria de apoio, os vigilante/porteiros apresentaram avaliação negativa mais evidente para o "Clima de trabalho em equipe" $(p=0,006)$, "Percepção da gerência do hospital" $(p=0,003)$ e "Condiçôes de trabalho" $(p=0,020)$. Entre os profissionais de nutrição/dietética sobressaiu-se a avaliação negativa para "Percepção da gerência da unidade" ( $\mathrm{p}=0,004)$. Em contraponto, os trabalhadores da manutenção apresentaram proporçôes mais elevadas para cultura positiva no domínio "satisfação no trabalho" ( $\mathrm{p}=0,010)$.

Tabela 1. Análise descritiva do escore geral do Questionário de Atitude de Segurança e seus domínios nas instituições hospitalares $(\mathrm{n}=2634)$

\begin{tabular}{|c|c|c|c|c|c|c|c|c|c|c|c|c|}
\hline \multirow{2}{*}{ SAQ e Domínios geral* } & \multirow[t]{2}{*}{$\mathrm{n} \dagger$} & \multirow{2}{*}{ Média } & \multirow{2}{*}{$\begin{array}{l}\text { Desvio } \\
\text { padrão }\end{array}$} & \multirow[t]{2}{*}{ CVP $\ddagger$} & \multicolumn{3}{|c|}{ Intervalo IQ ${ }^{\S}$} & \multirow[t]{2}{*}{ Mínimo } & \multirow[t]{2}{*}{ Máximo } & \multicolumn{3}{|c|}{$\begin{array}{l}\text { Alfa de Cronbach por } \\
\text { Categoria profissional }\end{array}$} \\
\hline & & & & & 25 & 50 & 75 & & & Geral* $^{*}$ & Saúde & Apoio \\
\hline SAQ total & 2.634 & 68,4 & 13,4 & 0,19 & 59,7 & 70,1 & 77,7 & 13,9 & 97,9 & 0,90 & 0,91 & 0,91 \\
\hline Clima de trabalho em equipe & 1989 & 74,3 & 16,6 & 0,22 & 62,5 & 75,0 & 87,5 & 12,5 & 100,0 & 0,61 & 0,60 & 0,65 \\
\hline Clima de segurança & 2149 & 68,5 & 17,5 & 0,25 & 57,1 & 71,4 & 82,1 & 3,5 & 100,0 & 0,65 & 0,66 & 0,64 \\
\hline Satisfação no trabalho & 2556 & 83,6 & 17,4 & 0,20 & 75,0 & 90,0 & 95,0 & 0,0 & 100,0 & 0,78 & 0,75 & 0,83 \\
\hline Percepção de estresse & 1959 & 62,6 & 28,1 & 0,44 & 43,7 & 68,7 & 87,5 & 0,0 & 100,0 & 0,79 & 0,79 & 0,77 \\
\hline \multicolumn{13}{|l|}{ Percepção da gerência } \\
\hline Unidade & 2200 & 63,3 & 21,8 & 0,34 & 50,0 & 66,6 & 79,1 & 0,0 & 100,0 & 0,73 & 0,72 & 0,75 \\
\hline Hospital & 2332 & 60,8 & 23,2 & 0,38 & 45,0 & 60,0 & 80,0 & 0,0 & 100,0 & 0,76 & 0,75 & 0,76 \\
\hline Condições de trabalho & 1862 & 63,1 & 26,7 & 0,42 & 41,6 & 66,6 & 83,3 & 0,0 & 100,0 & 0,71 & 0,71 & 0,70 \\
\hline
\end{tabular}


Tabela 2. Distribuição das pontuações nos domínios do Questionário de Atitude de Segurança e cultura de segurança, de acordo com as categorias profissionais da área da Saúde e do Apoio

\begin{tabular}{|c|c|c|c|c|c|c|c|}
\hline \multirow{3}{*}{$\begin{array}{l}\text { Domínio do SAQ } \\
\text { Categoria profissional }\end{array}$} & \multirow{2}{*}{\multicolumn{5}{|c|}{ Pontuação do SAQ }} & \multicolumn{2}{|c|}{ Cultura de Segurança } \\
\hline & & & & & & \multirow{2}{*}{$\begin{array}{c}\text { Baixa } \\
n(\%)\end{array}$} & \multirow{2}{*}{$\begin{array}{l}\text { Alta* } \\
\mathrm{n}(\%)\end{array}$} \\
\hline & Média & DP & Mediana & Mínimo & Máximo & & \\
\hline \multicolumn{8}{|c|}{ Clima de trabalho em equipe $†$} \\
\hline Saúde & 75,6 & 15,9 & 79,1 & 16,6 & 100,0 & $648(39,8)$ & $981(60,2)$ \\
\hline Apoio & 69,5 & 19,1 & 70,8 & 16,6 & 100,0 & $193(54,4)$ & $162(45,6)$ \\
\hline \multicolumn{8}{|l|}{ Clima de segurançał } \\
\hline Saúde & 68,7 & 17,2 & 71,4 & 3,5 & 100,0 & $915(55,5)$ & $733(44,5)$ \\
\hline Apoio & 67,8 & 17,1 & 67,8 & 25,0 & 100,0 & $278(56,3)$ & $216(43,7)$ \\
\hline \multicolumn{8}{|l|}{ Satisfação no trabalho† } \\
\hline Saúde & 84,6 & 15,8 & 90,0 & 5,0 & 100,0 & $324(18,2)$ & $1461(81,8)$ \\
\hline Apoio & 80,4 & 22,5 & 90,0 & 0,0 & 100,0 & $189(24,7)$ & $575(75,3)$ \\
\hline \multicolumn{8}{|c|}{ Percepção de estresse trabalhoł } \\
\hline Saúde & 62,5 & 28,1 & 62,5 & 0,0 & 100,0 & $869(54,7)$ & $720(45,3)$ \\
\hline Apoio & 58,5 & 28,1 & 62,5 & 0,0 & 100,0 & $200(54,9)$ & $164(45,1)$ \\
\hline \multicolumn{8}{|c|}{ Percepção da gerência unidade $†$} \\
\hline Saúde & 62,1 & 21,3 & 66,6 & 0,0 & 100,0 & $1068(65,3)$ & $568(34,7)$ \\
\hline Apoio & 64,9 & 20,7 & 66,6 & 4,1 & 100,0 & $318(57,0)$ & $240(43,0)$ \\
\hline \multicolumn{8}{|c|}{ Percepção da gerência hospital† } \\
\hline Saúde & 59,1 & 22,8 & 60,0 & 0,0 & 100,0 & $1149(68,4)$ & $530(31,6)$ \\
\hline Apoio & 61,3 & 22,9 & 60,0 & 5,0 & 100,0 & $379(58,7)$ & $267(41,3)$ \\
\hline \multicolumn{8}{|l|}{ Condições de trabalhoł } \\
\hline Saúde & 63,1 & 27,0 & 66,6 & 0,0 & 100,0 & $847(54,6)$ & $705(45,4)$ \\
\hline Apoio & 65,4 & 24,1 & 66,6 & 0,0 & 100,0 & $172(56,0)$ & $135(44,0)$ \\
\hline
\end{tabular}

*Ponto de corte da dicotomização: pontuação $\geq 75$ pontos; †Teste U de Mann-Whitney p-valor $<0,001$; ¥ Teste U de Mann-Whitney $p$-value $>0,05$

Tabela 3. Avaliação da cultura de segurança de acordo com as categorias profissionais e domínios do Questionário de Atitude de Segurança $(n=2634)$

\begin{tabular}{|c|c|c|c|c|c|c|c|c|c|c|c|c|c|c|c|}
\hline \multirow{5}{*}{ Categoria profissional } & \multirow{5}{*}{$\mathrm{n}$} & \multicolumn{14}{|c|}{ Domínios da Cultura de Segurança (SAQ) } \\
\hline & & \multirow{2}{*}{\multicolumn{2}{|c|}{$\begin{array}{l}\text { Clima trabalho } \\
\text { equipe }\end{array}$}} & \multirow{2}{*}{\multicolumn{2}{|c|}{$\begin{array}{l}\text { Clima de } \\
\text { segurança }\end{array}$}} & \multirow{2}{*}{\multicolumn{2}{|c|}{$\begin{array}{l}\text { Satisfação no } \\
\text { trabalho }\end{array}$}} & \multirow{2}{*}{\multicolumn{2}{|c|}{$\begin{array}{l}\text { Percepção do } \\
\text { estresse }\end{array}$}} & \multirow{2}{*}{\multicolumn{4}{|c|}{$\begin{array}{l}\text { Percepção da gerência } \\
\text { Unidade } \quad \text { Hospital }\end{array}$}} & \multirow{2}{*}{\multicolumn{2}{|c|}{$\begin{array}{l}\text { Condições de } \\
\text { trabalho }\end{array}$}} \\
\hline & & & & & & & & & & & & & & & \\
\hline & & Baixo & Alto* & Baixo & Alto & Baixo & Alto & Baixo & Alto & Baixo & Alto & Baixo & Alto & Baixo & Alto \\
\hline & & \multicolumn{2}{|c|}{$\%$} & \multicolumn{2}{|c|}{$\%$} & \multicolumn{2}{|c|}{$\%$} & \multicolumn{2}{|c|}{$\%$} & \multicolumn{2}{|c|}{$\%$} & \multicolumn{2}{|c|}{$\%$} & \multicolumn{2}{|c|}{$\%$} \\
\hline Categoria da Saúde & 1102 & 38,3 & 61,7 & 57,2 & 42,8 & 17,8 & 82,2 & 63,4 & 36,6 & 68,8 & 31,2 & 71,1 & 28,9 & 55,4 & 44,6 \\
\hline Enfermeiro & 339 & 45,7 & 54,3 & 58,6 & 41,4 & 20,9 & 79,1 & 49,9 & 50,1 & 65,2 & 34,8 & 67,3 & 32,7 & 63,7 & 34,3 \\
\hline Médico & 113 & 25,2 & 74,8 & 59,8 & 40,2 & 23,9 & 76,1 & 40,7 & 59,3 & 68,9 & 31,1 & 71,8 & 28,2 & 79,3 & 20,7 \\
\hline Farmacêutico & 52 & 47,4 & 52,6 & 57,7 & 42,3 & 17,3 & 82,7 & 67,3 & 32,7 & 61,2 & 38,8 & 68,6 & 31,4 & 67,3 & 32,7 \\
\hline Fisioterapeuta & 11 & 10,0 & 90,0 & 18,2 & 81,8 & 9,1 & 90,9 & 54,5 & 45,5 & 30,0 & 70,0 & 54,5 & 45,5 & 27,3 & 72,7 \\
\hline Nutricionista & 21 & 42,9 & 57,1 & 66,7 & 33,3 & 33,3 & 66,7 & 76,2 & 23,8 & 78,9 & 21,1 & 71,4 & 28,6 & 61,9 & 38,1 \\
\hline Psicólogo & 7 & 25,0 & 75,0 & 28,6 & 71,4 & 14,3 & 85,7 & 71,4 & 28,6 & 80,0 & 20,0 & 57,1 & 42,9 & 83,3 & 16,7 \\
\hline Dentista & 6 & 33,3 & 66,7 & 20,0 & 80,0 & --- & 100 & 83,3 & 16,7 & 33,3 & 66,7 & 16,7 & 83,3 & 33,3 & 66,7 \\
\hline Outros & 178 & 53,3 & 46,7 & 70,1 & 29,9 & 25,3 & 74,7 & 69,0 & 31,0 & 63,6 & 36,4 & 75,4 & 24,6 & 74,7 & 25,3 \\
\hline Total & 1830 & 39,8 & 60,2 & 58,6 & 41,4 & 19,5 & 80,5 & 60,4 & 39 & 67,2 & 32,8 & 70,4 & 29,6 & 60,8 & 39,2 \\
\hline$p$-value $\dagger$ & & \multicolumn{2}{|c|}{0,026} & \multicolumn{2}{|c|}{0,012} & \multicolumn{2}{|c|}{0,025} & \multicolumn{2}{|c|}{0,115} & \multicolumn{2}{|c|}{0,111} & \multicolumn{2}{|c|}{0,592} & \multicolumn{2}{|c|}{0,001} \\
\hline \multicolumn{16}{|l|}{ Categoria do Apoio } \\
\hline Administrativo & 328 & 53,1 & 46,9 & 58,5 & 41,5 & 24,3 & 75,7 & 54,6 & 45,4 & 60,6 & 39,4 & 62,3 & 37,7 & 57,5 & 42,5 \\
\hline $\begin{array}{l}\text { Serviço de higiene e } \\
\text { limpeza }\end{array}$ & 174 & 63,9 & 36,1 & 52,9 & 47,1 & 30,5 & 69,5 & 48,1 & 51,9 & 49,7 & 50,3 & 57,7 & 42,3 & 48,4 & 51,6 \\
\hline Nutrição/dietética & 92 & 50,0 & 50,0 & 60,6 & 39,4 & 37,5 & 62,5 & 59,2 & 40,8 & 72,3 & 27,7 & 70,0 & 30,0 & 67,4 & 32,6 \\
\hline Manutenção & 34 & 53,8 & 46,2 & 50,0 & 50,0 & 5,9 & 94,1 & 55,0 & 45,0 & 42,9 & 57,1 & 44,4 & 55,6 & 28,6 & 71,4 \\
\hline Vigilante/porteiro & 29 & 88,2 & 11,8 & 70,0 & 30,0 & 32,0 & 68,0 & 90,0 & 10,0 & 69,6 & 30,4 & 76,2 & 23,8 & 85,7 & 14,3 \\
\hline Chefias/diretores & 10 & 16,7 & 83,3 & 57,1 & 42,9 & 20,0 & 80,0 & 80,0 & 20,0 & 50,0 & 50,0 & 37,5 & 62,5 & 25,0 & 75,0 \\
\hline Outros & 137 & 42,1 & 57,9 & 50,0 & 50,0 & 14,1 & 85,9 & 55,0 & 45,0 & 47,1 & 52,9 & 44,3 & 55,7 & 53,1 & 46,9 \\
\hline Total & 804 & 54,1 & 45,9 & 56,2 & 43,8 & 24,7 & 75,3 & 54,8 & 45,2 & 37,0 & 43,0 & 58,6 & 41,4 & 56,0 & 44,0 \\
\hline$p$-value $†$ & & \multicolumn{2}{|c|}{0,006} & \multicolumn{2}{|c|}{0,606} & \multicolumn{2}{|c|}{0,010} & \multicolumn{2}{|c|}{0,353} & & & & & & \\
\hline
\end{tabular}

*Ponto de corte em todos os domínios: pontuação $\geq 75$; † Teste Qui-Quadrado 


\section{Discussão}

A taxa geral de respostas à pesquisa foi adequada, tendo em vista a população e a temática estudada, quando comparado às demais publicaçôes. ${ }^{(4,7,10)} \mathrm{O}$ percentual de respostas foi inferior a estudo português ${ }^{(10)}$ e árabe, ${ }^{(11)}$ mas superior a pesquisas brasileiras, ${ }^{(4,7,12)}$ contexto em que ainda são recentes as discussóes sobre cultura de segurança. A confiabilidade do instrumento mostrou-se satisfatória, corroborando outras evidências na avaliação geral entre 0,83 e 0,86 . $^{(8,12,13)}$

A avaliação geral de cultura de segurança do paciente apresentou predominância negativa entre as instituiçóes pesquisadas. Este achado assemelha-se a pesquisas internacionais, desenvolvidas na China ${ }^{(14)}$ e na Palestina, ${ }^{(15)}$ com escore variando de $61,3^{(15)}$ a $70,2^{(14)}$ e a estudos nacionais realizados no Ceará, ${ }^{(4,7)}$ com pontuaçóes entre 63,4 e 71,5..$^{(4,7)}$ No entanto, difere de estudo com trabalhadores do apoio realizado no mesmo estado, que apresentou cultura de segurança positiva. ${ }^{(12)}$

Identificaram-se percepçóes positivas, em ambas as categorias profissionais, no domínio "Satisfação no trabalho", que obteve maior pontuação quando comparado aos demais domínios avaliados, principalmente entre os dentistas. Assemelhou-se a estudos internacionais ${ }^{(16,17)}$ e nacionais que apresentaram escores favoráveis. ${ }^{(4,7,12,18)}$ Salienta-se a relevância da percepção positiva para esse domínio, visto que a qualidade da assistência prestada pelos profissionais e por conseguinte a segurança do paciente, tem relação direta com a satisfação dos mesmos. ${ }^{(18)}$ Aspectos como reconhecimento, gostar da atuação profissional e o bom relacionamento no ambiente laboral, contribuem para a satisfação no trabalho. ${ }^{(19)}$

Outrossim, estudo chinês, desenvolvido em uma unidade pediátrica, corrobora os achados positivos encontrados no domínio de "Clima de trabalho em equipe". (20) O trabalho em equipe realizado por meio de uma relação que proporciona conhecimento, motivação, colaboração, interação e cooperação entre os profissionais, contribui para a redução de eventos adversos e reduz taxas de complicaçóes referentes ao cuidado prestado. ${ }^{(13,19)}$ Outro aspecto importante é a comunicação eficiente e constante entre a gerência e os demais profissionais. ${ }^{(13)}$ Assim, o clima de trabalho em equipe positivo contribui para a promoção de um ambiente saudável, o qual possibilita a realização de cuidados seguros e, consequentemente, fortalece a cultura de segurança. ${ }^{(20)}$

Ao analisar o "Clima de trabalho em equipe" entre as categorias profissionais, evidenciou-se que os fisioterapeutas apresentaram resultados positivos superiores aos demais, principalmente quando comparado aos vigilantes/porteiros, que o avaliaram negativamente. A autonomia e a dinâmica de trabalho distinto do fisioterapeuta, nas atividades laborais, pode ser o indicativo do excelente relacionamento deste com as demais equipes. No que tange aos trabalhadores do serviço de apoio, estudo corrobora as evidências de que os mesmos apresentam escores mais baixos para este domínio e são menos favoráveis a atitudes de segurança do que aqueles que prestam o cuidado direto. ${ }^{(12)}$ Embora, a atividade, por vezes solitária, pode desencadear a percepção de ausência de companheirismo nas relaçóes de trabalho.

Trabalhadores de apoio desenvolvem atividades essenciais para a assistência segura e com qualidade ao paciente. Entretanto, vivenciam sentimentos de desvalorização e desprezo em relação ao desenvolvimento de suas atividades, interferindo diretamente na satisfação laboral. ${ }^{(21)}$ Acredita-se que isso ocorra pela falta de integração e fortalecimento entre as equipes, distanciando-os do processo de cuidado ao paciente e demonstrando a necessidade de estímulo à educação permanente, e a inserção transversal do tema como estratégias para a construção da cultura da segurança do paciente. ${ }^{(22)}$

Nesta perspectiva, aponta-se que as relaçóes interpessoais carecem de aprimoramento nas instituiçôes de saúde, em especial, entre os profissionais do serviço de apoio. Estudos ${ }^{(23,24)}$ apontam que as diferentes percepçóes podem variar de acordo com o cargo. Essas variaçóes, segundo pesquisa realizada na Arábia Saudita, provavelmente estão relacionadas a diferenças de status/autoridade, responsabilidades e capacitações diferenciais, questôes de gênero e as culturas profissionais distintas. ${ }^{(24)}$

Quanto ao domínio "Clima de segurança”, a avaliação geral foi negativa, contrapondo-se a pesquisa desenvolvida com 630 trabalhadores da saúde e do apoio. ${ }^{(12)}$ Embora, neste, observou-se associação entre fisioterapeutas e percepção positiva de segurança. A relevância deste domínio está na sua relação com 
a segurança do paciente, uma vez que hospitais com níveis mais altos de clima de segurança apresentam menor incidência de complicaçôes evitáveis e eventos adversos. ${ }^{(25)}$ Além disso, os profissionais dos cargos de apoio apresentaram os mais baixos escores neste domínio. Estudo aponta que o fato do trabalhador ter a possibilidade de escolher sua unidade de atuação, tem associação da percepçáo positiva quanto ao clima de segurança, configurando-se como uma estratégia de fortalecimento da cultura. ${ }^{(12)}$

Em relação a "Percepção da gerência da unidade e do hospital”, observou-se uma visão geral negativa referente às açóes voltadas à segurança do paciente. Cabe destacar que a gerência apresenta papel fundamental de planejar, elaborar, instituir e monitorar ações voltadas a promoção da cultura organizacional, sensibilizando os profissionais da equipe sobre o cuidado seguro. ${ }^{(26)}$ Para que haja fortalecimento da cultura de segurança, uma gestão participativa é indispensável para conhecer e auxiliar nos desafios que a equipe enfrenta. Por meio de uma comunicação efetiva, a participação dos gestores nas atividades, pode contribuir para uma relação de confiança entre todos os profissionais. ${ }^{(27)}$

Nas associaçóes, constatou-se que vigilantes/ porteiros juntamente com trabalhadores da nutrição/dietética, têm uma percepção negativa da gerência da unidade e do hospital. Esses achados estão em oposição aos padróes positivos detectados na avaliação dos trabalhadores da manutenção para esses domínios, bem como quando comparados a outro estudo $^{(12)}$ com população semelhante, o que sugere uma maior aproximação da chefia com sua equipe de trabalho e esta com seus superiores. Assim, sinaliza-se a necessidade de readequação nos processos de trabalho nas instituiçóes de saúde, e a inclusão de todas categorias profissionais na elaboração de estratégias de melhoria e decisóes administrativas.

Ainda, os profissionais de saúde e dos serviços de apoio, em especial, psicólogos e vigilantes/porteiros, apresentaram escores negativos a respeito do domínio "Condiçóes de trabalho", condizente com achados de uma pesquisa no nordeste do Brasil. ${ }^{(18)}$ Evidencia-se que aspectos relacionados a falta de qualificação, baixa remuneração, número reduzido de profissionais, exposição a fatores de risco, contribuem para con- dições de trabalho inadequadas. ${ }^{(27)}$ Neste contexto, ressalta-se a importância em investigar e promover ambientes favoráveis a realização das atividades profissionais, uma vez que este influencia diretamente na qualidade da assistência prestada. ${ }^{(18,27)}$

A partir do exposto, entende-se como limitação do estudo, a dificuldade em confrontar dados relacionados aos profissionais do serviço de apoio, pela escassez de produçôes científicas com essa população. ${ }^{(12)}$ Sabese que, embora ainda permaneçam com pouca visibilidade e, às vezes, não percebam sua real importância como integrantes de uma equipe, estes profissionais representam um papel significativo nas instituiçôes, pois prestam o cuidado indireto ao paciente e auxiliam as demais categorias na excelência da assistência prestada.

Ademais, a variabilidade do porte institucional e complexidade dos hospitais avaliados podem ter interferido na interpretação dos resultados. Entende-se, em concordância com as evidências científicas, ${ }^{(25)}$ que os contextos institucionais, assim como as unidades dentro de um mesmo hospital, além de uma cultura geral de segurança do paciente, se organizam e criam subculturas de acordo com suas especificidades.

\section{Conclusão}

A cultura de segurança do paciente foi avaliada como negativa pelos trabalhadores, exceto nos domínios "Satisfação no trabalho" e "Clima de trabalho em equipe". A "Percepção de gerência do hospital" obteve resultados mais baixos e esse indicador pode estar relacionado a problemas gerenciais reais, que interferem na motivação dos trabalhadores. Quando comparadas as categorias profissionais (saúde e apoio), identificou-se pouca variabilidade nos escores. No entanto, os profissionais do serviço de apoio tendem a escores mais baixos. Os fisioterapeutas, dentistas e trabalhadores da manutenção avaliaram de forma positiva a cultura de segurança. Já psicólogos, profissionais da nutrição/dietética e vigilantes/porteiros tiveram maiores percentuais para cultura negativa. A mudança de cultura em relação à segurança deve partir do envolvimento da gestão, na revisão e melhoria dos processos de trabalho, que podem repercutir no comportamento e desempenho dos demais trabalhadores. 


\section{Agradecimentos}

\author{
Edital 011/2014 PIBIC - Programa Institucional \\ de Bolsas de Iniciação Científica (CNPq/UFSM). \\ Edital 010/2014 PROIC HUSM - Programa de \\ Iniciação Científica para o Hospital Universitário \\ de Santa Maria.
}

\section{Colaborações}

Baratto MAM, Seidel EJ, Morais BX, Munhoz OL, Silva SC, Zottele C e Magnago TSBS contribuíram com a concepçáo do estudo, análise e interpretação dos dados, redação do artigo, revisão crítica relevante do conteúdo intelectual e aprovaçáo final da versão a ser publicada.

\section{Referências}

1. Petitta L, Probst TM, Barbaranelli C, Ghezzi V. Disentangling the roles of safety climate and safety culture: multi-level effects on the relationship between supervisor enforcement and safety compliance. Accid Anal Prev. 2017;99 Pt A:77-89.

2. de Carvalho RE, Cassiani SH. Cross-cultural adaptation of the Safety Attitudes Questionnaire - Short Form 2006 for Brazil. Rev Lat Am Enfermagem. 2012;20(3):575-82.

3. Agência Nacional de Vigilância Sanitária (ANVISA). Resolução da Diretoria Colegiada da ANVISA- RDC nº 36 de 35 de julho de 2013. Institui ações para a segurança do paciente em serviços de saúde e dá outras providências. Diário Oficial da União; 2013. Disponível em: $\quad$ http://bvsms.saude.gov.br/bvs/saudelegis/anvisa/2013/ rdc0036_25_07_2013.html

4. Carvalho RE, Arruda LP, Nascimento NK, Sampaio RL, Cavalcante ML, Costa AC. Assessment of the culture of safety in public hospitals in Brazil. Rev Lat Am Enfermagem. 2017;25:e2849.

5. Nordén-Hägg A, Sexton JB, Kälvemark-Sporrong S, Ring L, Kettis-Lindblad $\AA$. Assessing safety culture in pharmacies: the psychometric validation of the Safety Attitudes Questionnaire (SAQ) in a national sample of community pharmacies in Sweden. BMC Clin Pharmacol. 2010;10(8):8.

6. Galvão TF, Lopes MC, Oliva CC, Araújo ME, Silva MT. Patient safety culture in a university hospital. Rev Lat Am Enfermagem. 2018;26(0):e3014.

7. Girão AL, Lacerda AJ, Carvalho LS, Lousada LM, Nascimento KM, Cruz $\mathrm{KT}$, et al. Cultura de segurança do paciente em unidades de terapia intensiva: percepção de profissionais de saúde. Rev Eletr Enferm. 2019;21:50649.

8. Santiago TH, Turrini RN. Organizational culture and climate for patient safety in Intensive Care Units. Rev Esc Enferm USP. 2015;49(Spec):121-7.

9. Sexton J, Thomas E, Grillo S: The Safety Attitudes Questionnaire (SAQ) guidelines for administration. Austin, The University of Texas Center of
Excellence for Patient Safety Research and Practice, 2003 (Technical Report 03/02/2008).

10. Fassarela CS, Silva LD, Camerini FG, Barbieri-Figueiredo MC. Indicador organizacional da cultura de segurança em um hospital universitário. Rev Enferm UERJ On line. 2019;27:e34073.

11. Al Nadabi W, Faisal M, Mohammed MA. Patient safety culture in Oman: A national study. J Eval Clin Pract. 2020;26(5):1406-1415.

12. Kolankiewicz AC, Schmidt CR, Carvalho RE, Spies J, Dal Pai S, Lorenzini E. Cultura de segurança do paciente na perspectiva de todos os trabalhadores de um hospital geral. Rev Gaúcha Enferm. 2020;41:e20190177.

13. Golle L, Ciotti D, Herr GE, Aozane F, Schmidt CT; Adriane Kolankiewicz AR. Cultura de segurança do paciente em hospital privado. Cuidado Fundamental; 2018;10(1):85-9.

14. Jiang K, Tian L, Yan C, Li Y, Fang H, Peihang S, et al. A cross-sectional survey on patient safety culture in secondary hospitals of Northeast China. PLoS One. 2019;14(3):e0213055.

15. Elsous A, Akbari Sari A, Rashidian A, Aljeesh Y, Radwan M, AbuZaydeh $\mathrm{H}$. A cross-sectional study to assess the patient safety culture in the Palestinian hospitals: a baseline assessment for quality improvement. JRSM Open. 2016;7(12):2054270416675235.

16. Soh SE, Morello R, Rifat S, Brand C, Barker A. Nurse perceptions of safety climate in Australian acute hospitals: a cross-sectional survey. Aust Health Rev. 2018;42(2):203-9.

17. Göras C, Unbeck M, Nilsson U, Ehrenberg A. Interprofessional team assessments of the patient safety climate in Swedish operating rooms: a cross-sectional survey. BMJ Open. 2017;7(9):e015607.

18. Oliveira IC, Cavalcante ML, Aires SF, Freitas RJ, Silva BV, Marinho DM, et al. Safety culture: perception of health professionals in a mental hospital. Rev Bras Enferm. 2018;71 Suppl 5:2316-22.

19. Toso GL, Golle L, Magnago TS, Herr GE, Loro MM, Aozane F, et al. Cultura de segurança do paciente em instituições hospitalares na perspectiva da enfermagem. Rev Gaúcha Enferm. 2016;37(4):e58662.

20. Huang CH, Wu HH, Lee YC, Van Nieuwenhuyse I, Lin MC, Wu CF. Patient safety in work environments: perceptions of pediatric healthcare providers in Taiwan. J Pediatr Nurs. 2020;53:6-13.

21. Fontoura FP, Gonçalves CG, Soares VM. Condições e ambiente de trabalho em uma lavanderia hospitalar: percepção dos trabalhadores. Rev Bras Saúde Ocup. 2016;41:e5.

22. Wegner W, Silva SC, Kantorski KJ, Predebon CM, Sanches MO, Pedro EN. Education for culture of patient safety: implications to professional training. Esc Anna Nery. 2016;20(3):e20160068.

23. Huang $\mathrm{CH}, \mathrm{Wu} \mathrm{HH}$, Lee YC. The perceptions of patient safety culture: A difference between physicians and nurses in Taiwan. Appl Nurs Res. 2018;40:39-44.

24. Alzahrani N, Jones R, Abdel-Latif ME. Attitudes of doctors and nurses toward patient safety within emergency departments of two Saudi Arabian hospitals. BMC health Serv Res. 2018;18: article number 736.

25. Soh SE, Morello R, Rifat S, Brand C, Barker A. Nurse perceptions of safety climate in Australian acute hospitals: a cross-sectional survey. Aust Health Rev. 2018;42(2):203-9.

26. Magalhães FH, Pereira ICA, Luiz RB, Barbosa MH, Ferreira MB. Clima de segurança do paciente em um hospital de ensino. Rev Gaúcha Enferm. 2019; 40(Esp):e20180272 .

27. Pavan NF, Magalhães AL, Poncio DF, Ascari RA, Zanini PD, Knihs NS, et al. Cultura de segurança do paciente no transplante renal no oeste catarinense. Acta Paul Enferm. 2019;32(4):398-405. 